\title{
TERAHERTZ SPECTROSCOPY USING QUANTUM-CASCADE LASERS
}

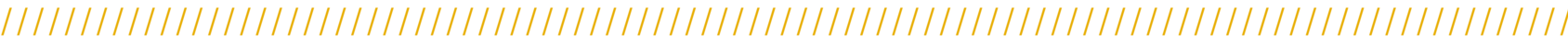

Heinz-Wilhelm HÜBERS ${ }^{1,2^{*}}$, Heiko RICHTER ${ }^{1}$, Martin WIENOLD ${ }^{1}$, Xiang LU ${ }^{3}$, Lutz SCHROTTKE ${ }^{3}$ and Holger T. GRAHN ${ }^{3}$

${ }^{1}$ German Aerospace Center (DLR), Institute of Optical Sensor Systems, Rutherfordstraße 2, 12489 Berlin, Germany

${ }^{2}$ Humboldt-Universität zu Berlin, Department of Physics, Newtonstr. 15, 12489 Berlin, Germany

${ }^{3}$ Paul-Drude-Institut für Festkörperelektronik, Leibniz-Institut im Forschungsverbund Berlin e. V., Hausvogteiplatz 5-7, 10117 Berlin, Germany

*Heinz-Wilhelm.Huebers@dlr.de
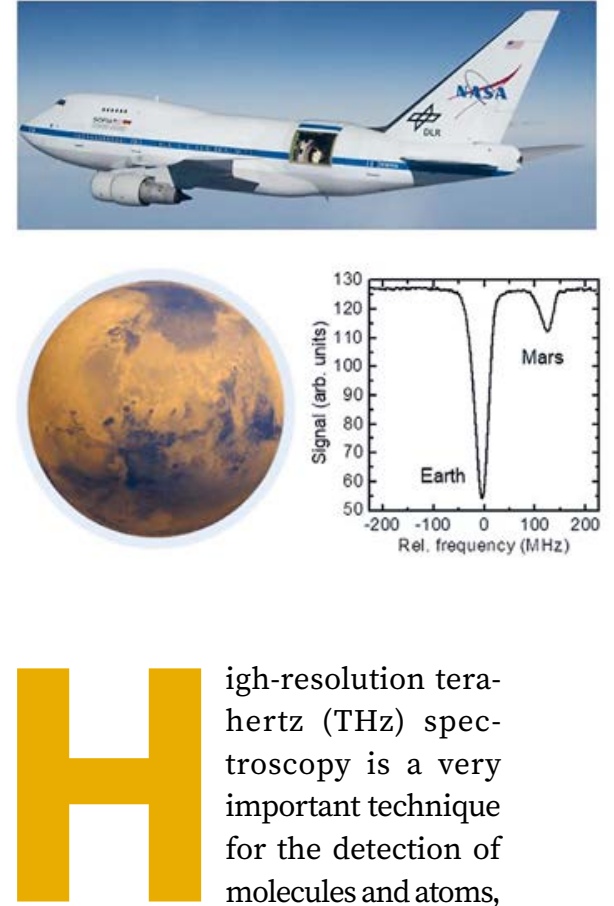

igh-resolution terahertz (THz) spectroscopy is a very important technique for the detection of molecules and atoms, since they exhibit characteristic absorption or emission spectra in the $\mathrm{THz}$ spectral region due to transitions between rotational states of molecules and fine-structure transitions within a multiplet of orbital angular momentum states in atoms. For example, molecules and atoms such as the hydroxyl radical $\mathrm{OH}$ and atomic oxygen can be detected in space or in the atmospheres of the Earth and other planets. The hydroxyl radical plays a key role in the chemistry of the stratosphere. It has rotational transitions at about 2.5 and 3.5 THz. Atomic oxygen, which is important for the chemistry and energy

\begin{abstract}
Terahertz (THz) quantum-cascade lasers (QCLs) provide powerful, narrow-band, and frequencytunable radiation, which makes them ideal sources for high-resolution molecular spectroscopy. A first application of a THz QCL has been as local oscillator in a heterodyne spectrometer for astronomy on board a Boeing 747. For laboratory spectroscopy, QCLs close the so-called THz gap and enable new research topics.
\end{abstract}

https://doi.org/10.1051/photon/202010127

balance of the mesosphere and lower thermosphere, can be detected through its fine-structure transition at about 4.7 THz. Furthermore, THz spectroscopy allows for the investigation of the structure and energy levels of molecules and atoms as well as for the determination of Doppler and pressure broadening.

Until the realization of $\mathrm{THz}$ quantum-cascade lasers (QCLs) in 2002 [1], high-resolution THz spectroscopy with a resolving power of $10^{6}$ or better was very difficult to achieve due to the lack of suitable radiation sources. Because of the very high spectral brightness of $\mathrm{THz}$ QCLs, which is typically on the order of several $\mathrm{mW}$ per $\mathrm{MHz}$, they became important for high-resolution molecular spectroscopy [2]. THz QCLs have been implemented in heterodyne as well as in absorption spectrometers. Heterodyne spectrometers are mostly used for remote sensing in astronomy and in atmospheric science. In this case, a very weak signal on a low-power background has to be detected. In a heterodyne spectrometer, the QCL serves as a local oscillator. In absorption spectroscopy, the goal is to detect a small fractional change of a relatively large amount of power. Although both applications are different, the requirements regarding the QCL are similar. Additional advantages of THz QCLs, covering at present the emission frequency range of about 1 to $5 \mathrm{THz}$, are compactness and optical output powers of typically several mW. A challenge is still the necessary cryogenic operation. However, mechanical coolers, which are free of cryogenic liquids, can be used. 


\section{TERAHERTZ QUANTUM- CASCADE LASERS: PHYSICS AND TECHNOLOGY}

The radiation of QCLs, which were invented by F. Capasso and his coworkers more than 25 years ago [3], is emitted by intersubband transitions within the conduction band rather than by transitions across the energy gap. The optically active regions, which contain the laser levels, are connected by extractor/injector stages forming a cascade structure as shown in Figure 1. The carrier transport through the structure relies on resonant tunneling between adjacent quantum wells and on carrier scattering between subbands. In order to achieve population inversion, a well-balanced interplay of scattering processes and stimulated emission has to be established. Both, the scattering rates and the emission wavelengths, can be tailored by varying the thickness of the individual layers.

THz QCLs are based on two main design types: bound-to-continuum and resonant-phonon designs [4]. A combination of these two types, the hybrid design (Fig. 1), is often preferred for continuous-wave operation. The rather complex heterostructures consist of a single period with typically 6 to 20 layers, which is repeated about 100 times. The thicknesses of the layers vary between a few $\mathrm{nm}$ and about $20 \mathrm{~nm}$ resulting in a total thickness of the QCL structure of typically about $10 \mu \mathrm{m}$. For the modeling of THz QCLs, which is necessary

a

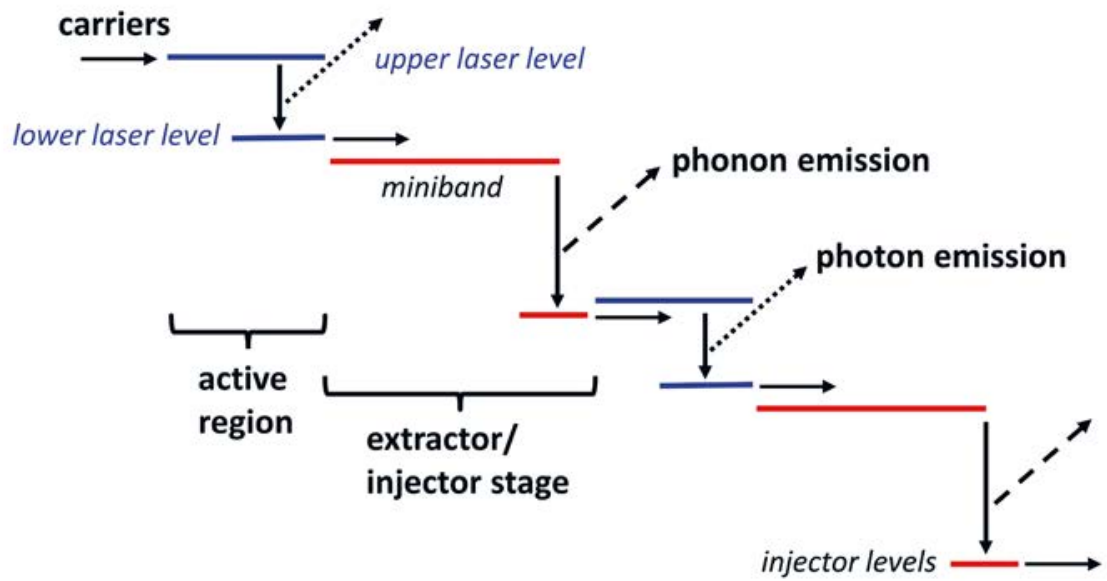

b

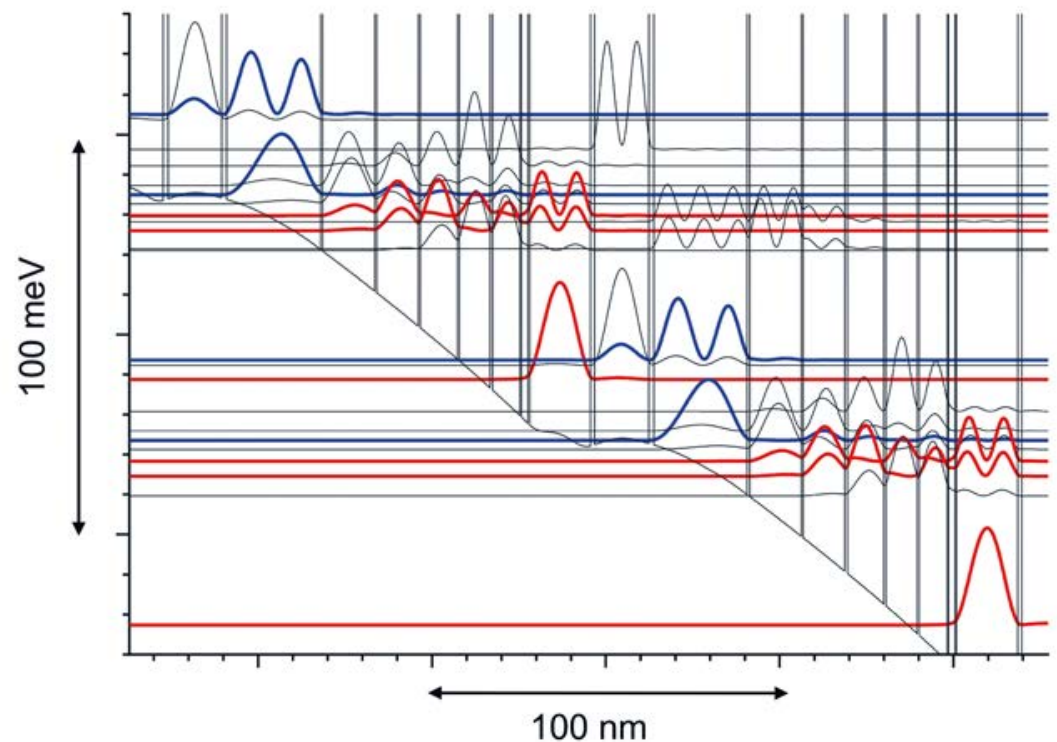

in order to develop advanced designs and to exploit new frequency ranges, several theoretical approaches have been employed. The complexity of the methods increases starting from most straightforward and fast rate equation systems over full density matrix models and Monte Carlo simulations to non-equilibrium Green's function theories with increasing physical insight, but also with significantly increasing numerical costs [5].

THz QCLs are mainly based on $\mathrm{GaAs} / \mathrm{Al}_{x} \mathrm{Ga}_{1-x} \mathrm{As}$ heterostructures with $0.1 \leq x \leq 0.25$, which are grown by molecular beam epitaxy with a very good stability of the growth parameters over up to 20 hours. In order to achieve the necessary long-term stability, in-situ growth rate control methods are employed. To form a laser waveguide, the wafers are processed using wet chemical or dry etching. Edge emitting Fabry-Pérot ridge lasers represent a straight-forward and robust approach. For single-mode operation, distributed-feedback lasers, two-section cavity lasers, or very short cavities are used. Examples for the waveguide and resonator configurations are presented in Figure 2.

Figure 1.

(a) Schematic representation and (b) subband structure (envelope functions) of a hybrid design for a QCL at 4.7 THz. The carriers (electrons) are injected from the left into the upper laser level. The emission of a $\mathrm{THz}$ photon is connected with the transition of a carrier to the lower laser level, which is coupled to a miniband (bound-to-continuum transition). After transport through the miniband, the electron emits a longitudinal optical phonon due to a transition resonant to the longitudinal optical phonon energy (resonantphonon transition) and is injected into the upper laser level of the following period of the cascade. In the boundto-continuum design, the transition resonant to the phonon energy is not present, while there is no miniband in the resonant-phonon design. 
Typical dimensions of the laser ridges are widths of 15 to $200 \mu \mathrm{m}$ and lengths of 0.5 to $7.5 \mathrm{~mm}$.

For continuous-wave operation in mechanical miniature cryocoolers, the wall plug efficiency of the lasers has to be sufficiently large for the limited available cooling power. For this case, we developed GaAs/AlAs THz QCLs, which exhibit an up to three times higher wall plug efficiency than $\mathrm{GaAs} / \mathrm{Al}_{0.25} \mathrm{Ga}_{0.75} \mathrm{As}$ QCLs with an almost identical active-region design. Currently, QCLs based on this alternative materials system are available for frequencies between 3.3 and $5.0 \mathrm{THz}$ (Fig. 3) [6].

\section{HIGH-RESOLUTION SPECTROSCOPY WITH TERAHERTZ QUANTUM-CASCADE LASERS}

THz heterodyne spectroscopy is the first example where THz QCLs found a unique application in astronomy. One particularly important example is the detection of atomic oxygen. It can be traced by measuring the emission from its fine-structure transition at 4.7 THz. THz heterodyne spectroscopy is the only technique which provides the required spectral resolution of around $1 \mathrm{MHz}$.

In a heterodyne spectrometer, a weak THz signal is detected by generating an intermediate frequency, which is the difference frequency of the signal radiation and the radiation from a local oscillator. The intermediate-frequency spectrum, which is a one-to-one replica of the THz spectrum, is recorded by a digital fast Fourier transform spectrometer (Fig. 4). Above about $3 \mathrm{THz}$, QCLs are the only radiation sources which comply with the requirements of a local oscillator with respect to an output power of several $\mathrm{mW}$, the transformation of the beam profile into an approximately Gaussian shape, and a well-defined, stable as well as tunable frequency. Since 2014, a THz QCL local oscillator [7] has routinely been used in the German Receiver for Astronomy at Terahertz Frequencies

\section{THZ TECHNOLOGIES}

(GREAT) on board the Stratospheric Observatory for Infrared Astronomy (SOFIA) for the observation of the atomic oxygen emission (Fig. 4). The QCL has a lateral distributed feedback grating, which is optimized for $4.745 \mathrm{THz}$ and allows for single-mode emission over most of the driving current range of the laser. Frequency stabilization on the order of $1 \mathrm{MHz}$ is achieved by a high-precision control of the driving current and heat sink temperature of the QCL. Besides for SOFIA, further QCL local oscillators are currently under development for future balloon or spaceborne missions.

In contrast, high-resolution absorption spectra are acquired by tuning the frequency of the QCL across the absorption line. The linewidth of the QCL radiation has to be significantly smaller than the absorption feature to be measured. The typical width of a Doppler-limited molecular rotational transition is a few MHz. With a sufficient temperature and driving current stabilization, the linewidth of a free running QCL is about $1 \mathrm{MHz}$, although this is still far above the fundamental quantum limit, which is on the order of several $10 \mathrm{~Hz}$ [9]. To improve the frequency stability and linewidth of the QCL, its radiation can be locked to a molecular reference line, to a reference source such as a microwave oscillator whose emission frequency is multiplied to the $\mathrm{THz}$ range, or to a frequency comb. With the latter two techniques, phase-locking has been demonstrated [10,11]. Very high frequency precision can also be obtained by injection locking to a telecom wavelength frequency comb [12]. Furthermore, if the frequency or wavelength of the QCL is modulated and the first or second harmonic is measured instead of the direct absorption signal, a minimum detectable absorption of better than $10^{-5}$ can be achieved.

The straightforward approach for frequency tuning is to change the driving current or operating temperature of the QCL, which allows for a
The new

generation

of interferometers

Di)

Your wavelength is our standard.

Get beyond $633 \mathrm{~nm}$ !

\section{More versatile}

Three interferometers for the price of one!

\section{More relevant}

Testing at $370 \mathrm{~nm}, 530 \mathrm{~nm}$, $1050 \mathrm{~nm}, 1550 \mathrm{~nm} . . . ?$ we have a standard solution for you

More cost-effective For UV, NIR, SWIR, LWIR versions

\section{More powerful}

High dynamic range up to $150 \mu \mathrm{m}$

\section{More robust}

Insensitive to vibration
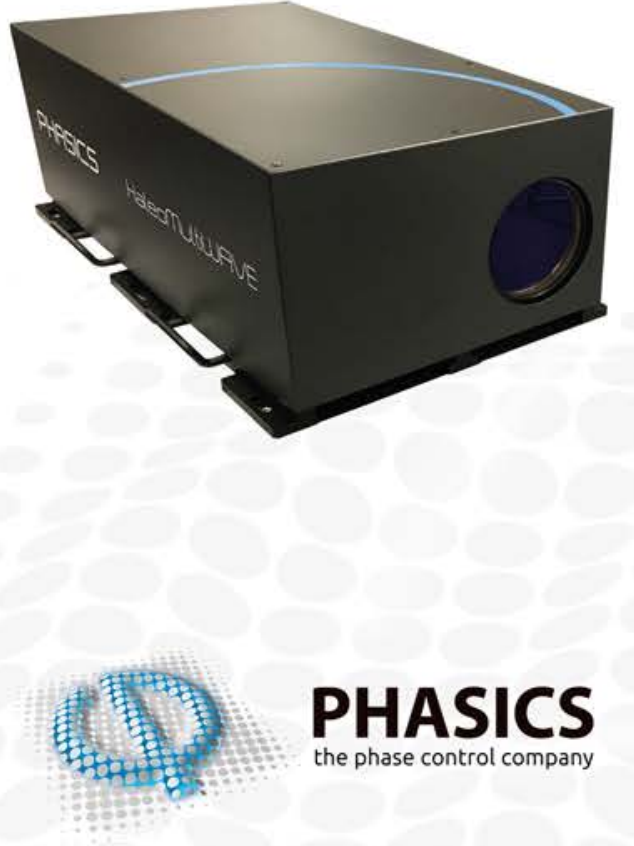
frequency tuning of several GHz. For larger tuning ranges, several techniques such as micro-optomechanical cavities, electronic frequency tuning in multi-terminal and multi-section QCLs, as well as tuning by gas adsorption have been demonstrated. However, all of these methods have particular shortcomings, which have prevented their use in high-resolution spectroscopy. Recently, frequency tuning has been demonstrated by illuminating the substrate below the active medium of the QCL with near-infrared radiation so that an electron-hole plasma in the vicinity of the back facet of the QCL is generated [13], which changes the optical length of its cavity. A tuning range of $40 \mathrm{GHz}$ has been achieved [14]. This method is rather straightforward, and its potential has been demonstrated by high-resolution spectroscopy of $\mathrm{CH}_{3} \mathrm{OH}$ [14] (Fig. 5). THz QCLs have also been employed for Lamb-dip spectroscopy, which requires the saturation of the transition, as well as for techniques based on optical feedback, which changes the QCL frequency as well as its terminal voltage. While in the first case the spectroscopic precision can be increased significantly, the latter allows for a much simpler spectrometer design, because the external detector can be omitted. Figure 5 shows a Lamb dip with a sub-Doppler linewidth of $170 \mathrm{kHz}$ for a rotational transition of HDO in a collinear pumpprobe configuration [15]. The feedback technique has been demonstrated with a mixture of $\mathrm{D}_{2} \mathrm{O}$ and $\mathrm{CH}_{3} \mathrm{OD}$ [16] as shown in Figure 5 by placing the absorption cell between the QCL and a mirror so that the radiation is reflected back into the QCL.

\section{OUTLOOK}

In the past few years, THz QCLs have reached a level of maturity which enables routine operation in various types of spectrometers. In particular, QCLs for local oscillators at frequencies above $3 \mathrm{THz}$ are currently unrivaled for heterodyne receivers in airborne instruments for astronomy and atmospheric research. Similar systems are now being developed for balloon-borne instruments and future space missions. For absorption spectroscopy using THz QCLs, many techniques as well as key performance parameters, which have already been established for other frequency ranges, have been

\section{Figure 2.}

Schematic representations of (a) a single-plasmon waveguide, in which the optical mode is confined between a top metal contact and a highly doped (contacting) layer underneath the cascade structure, and (b) a metal-metal waveguide, in which the radiation is confined between two metal layers. While metal-metal waveguides exhibit a larger mode confinement factor than single-plasmon waveguides and are preferred for high-temperature operation, single-plasmon waveguides allow for a better beam profile and larger output powers for rather straightforward resonator designs and are often preferred for practical applications. (c) Schematic layout for a THz QCL based on a single-plasmon waveguide. Since in this case the laser mode penetrates into the substrate, semi-insulating substrates are used so that the bottom contact formed by the highly doped layer is also accessed from the top. (d) and (e) present schematic views and scanning-electron micrographs of laser stripes with a lateral distributed-feedback grating fabricated by dry etching and a two-section resonator fabricated by focused-ion beam cuts, respectively, for single-mode operation.

a

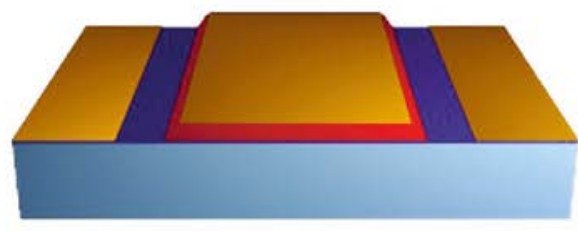

b

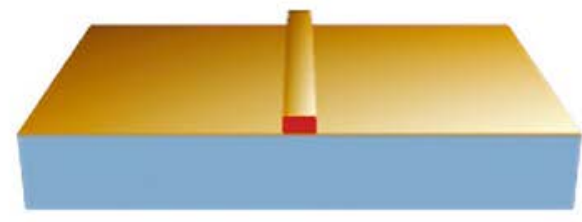

C

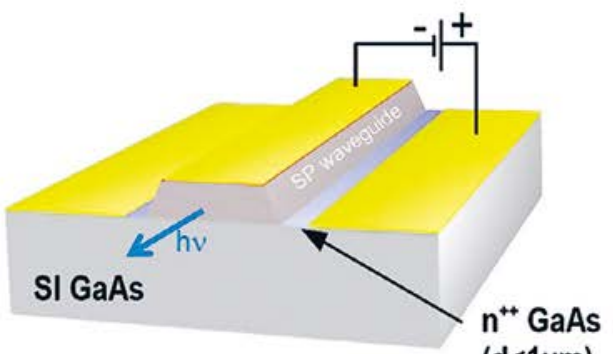

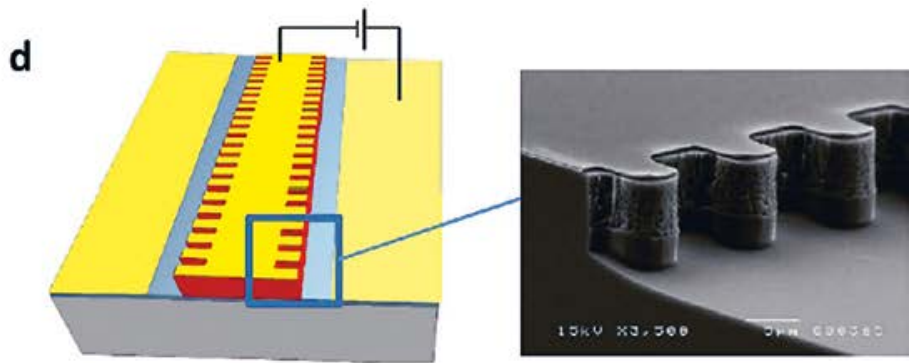

e

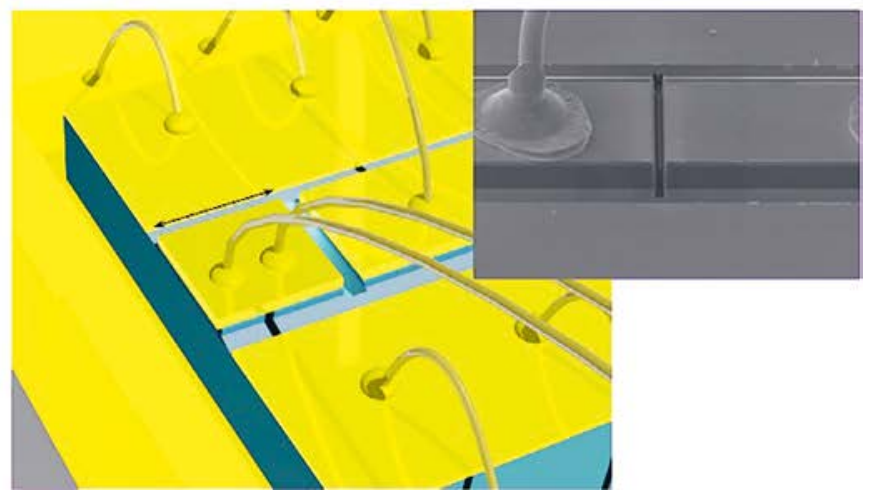


Emission wavenumber $\left(\mathrm{cm}^{-1}\right)$

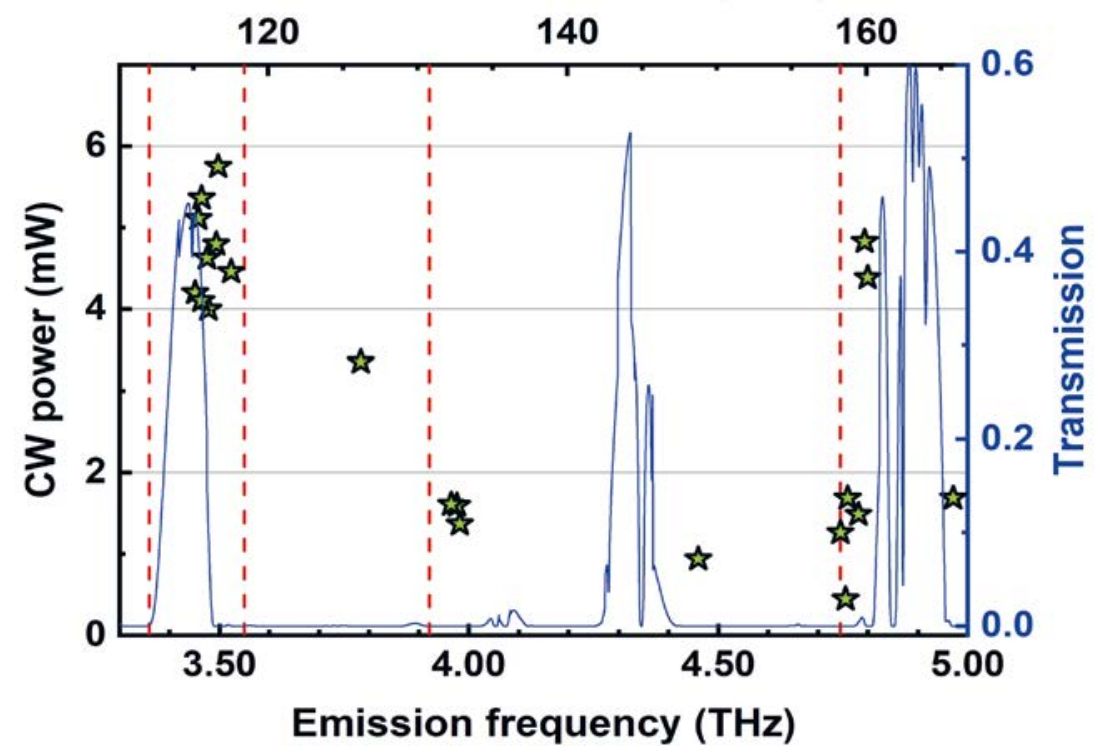

Figure 3.

Compilation of the optical properties of $21 \mathrm{GaAs} / \mathrm{AlAs} \mathrm{THz}$ QCLs based on the hybrid design. The asterisks depict the maximum output power for continuous-wave operation as a function of the center emission frequency (wavenumber) measured at a heat sink temperature of $30 \mathrm{~K}$. The vertical dashed lines indicate target frequencies of 3.36, 3.92, and $4.75 \mathrm{THz}$ for fine-structure transitions of $\mathrm{Al}, \mathrm{N}^{+}$, and $\mathrm{O}$ atoms/ions, respectively, and $3.55 \mathrm{THz}$ for $\mathrm{OH}$ detection. The blue solid line depicts a simulated transmission spectrum of air based on the HITRAN database for ambient conditions and an optical path length of $10 \mathrm{~m}$, exhibiting maxima at 3.43, 4.32, and 4.92 THz (adapted from Ref. [6]).

\section{Figure 4.}

GREAT heterodyne spectrometer aboard the airborne observatory SOFIA. The radiation from the QCL local oscillator is mixed with the 4.7-THz signal from the atmosphere of Mars and down-converted to a $\mathrm{GHz}$ frequency band as shown on the right. In the spectrum, an absorption feature due to atomic oxygen in the atmosphere of Mars appears together with one due to absorption in the mesosphere and lower thermosphere of the Earth. Due to the relative velocity of Mars and Earth, the absorption in the atmosphere of Mars is shifted by $140 \mathrm{MHz}$ from the absorption in the atmosphere of the Earth at $4.7448 \mathrm{THz}$ (rel. frequency: $0 \mathrm{MHz}$ ) (spectrum adapted from Ref. [8]).

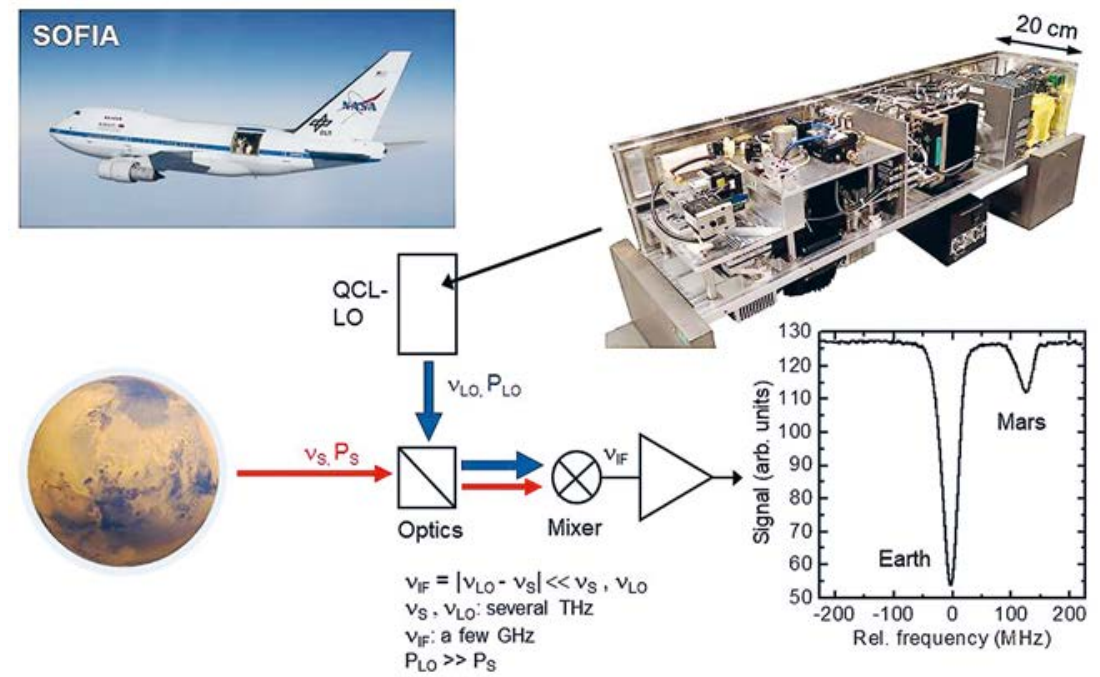

\section{New range of ultra-fast fiber lasers}

at exotic wavelengths!

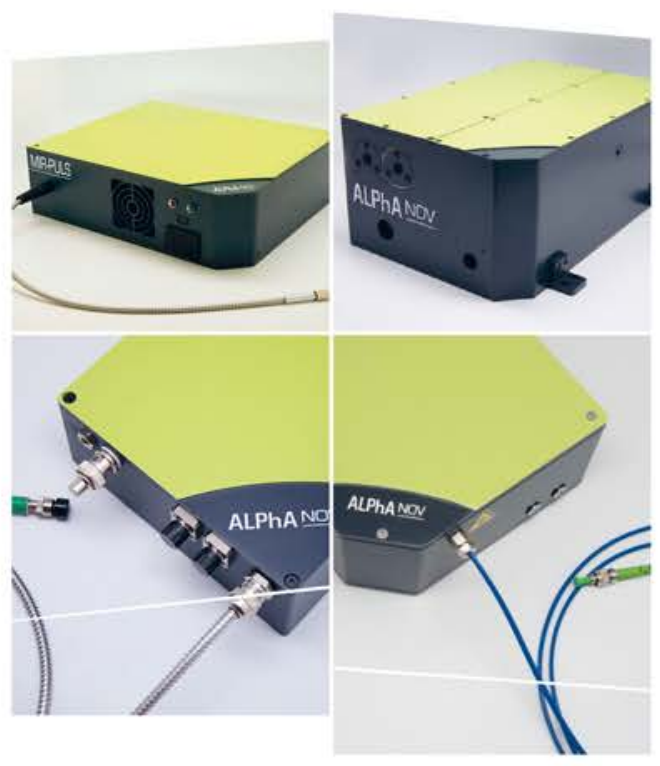

Ti-PULS: New generation of sub-40 fs fiber laser at $800 \mathrm{~nm}$

MIR-PULS: Tunable fiber laser sources sub $300 \mathrm{fs}$; $1800-2100 \mathrm{~nm}$

\section{Large range of compact oscillators:}

" Working at $1064 \mathrm{~nm}$ in picosecond regime [Yb-PULS-ps]

$=$ Working at $1550 \mathrm{~nm}$ in femtosecond regime

\section{MORE TO COME... \\ ALPhA NOV \\ Optics \& Lasers Technology Center}

YOUR PARTNER IN LASER \& OPTICS DEVELOPMENT AND INNOVATION 
demonstrated paving the way for further applications. In the years to come, THz QCLs are expected to enable exciting discoveries in molecular spectroscopy as well as in astronomy and atmospheric science.

Figure 5

(a) Absorption spectrum of $\mathrm{CH}_{3} \mathrm{OH}$ at $3.3 \mathrm{THz}$ (red line) measured by tuning the frequency of the QCL by illumination of its back facet with a near-infrared laser. The black line indicates a calculated spectrum using the HITRAN data base (adapted from Ref. [14]). (b) A 170-kHzwide Lamb-dip (full width at half maximum) of a transition in $\mathrm{HDO}$ which amounts to three times the theoretical value. The spectrum has been measured within only $200 \mathrm{~ms}$ at a gas pressure of $2.5 \mu$ bar (adapted from Ref. [15]). (c) Peak absorption of $\mathrm{D}_{2} \mathrm{O}$ (blue dots) and $\mathrm{CH}_{3} \mathrm{OD}$ (red dots) as a function of time obtained from the absorption spectra recorded by the optical feedback technique. Each 100-MHz wide spectrum, which covers one transition from both species (inset), takes $10 \mathrm{~ms}$ to be measured. Initially, the absorption cell contained only $\mathrm{D}_{2} \mathrm{O}$, while $\mathrm{CH}_{3} \mathrm{OD}$ was added slowly (adapted from Ref. [16]). (a) Frequency $(\mathrm{THz})$

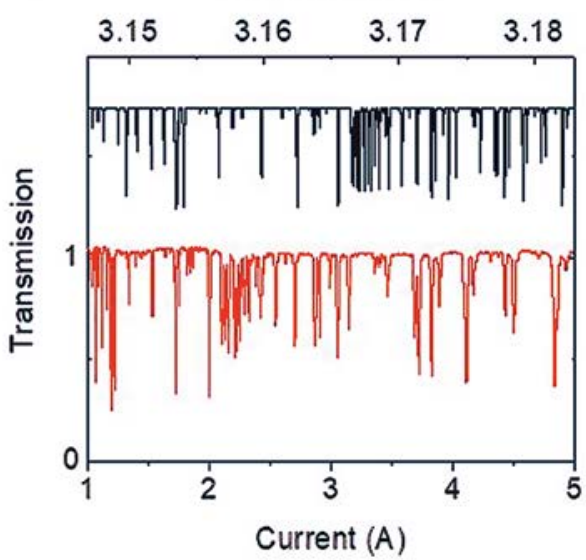

(b) Rel. frequency $(\mathrm{MHz})$

(c)

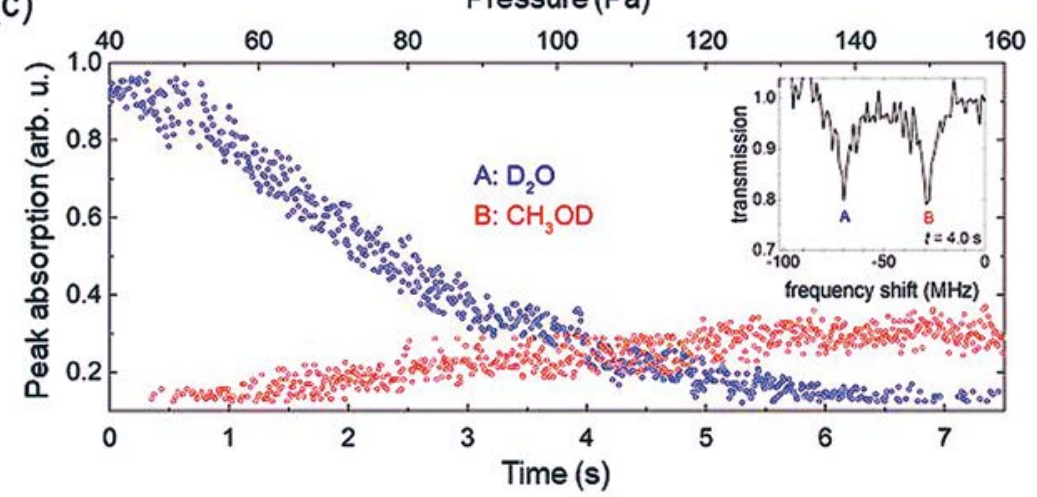

\section{REFERENCES}

[1] R. Köhler et al., Terahertz semiconductor-heterostructure laser, Nature 417, 156 (2002)

[2] H.-W. Hübers et al., High-resolution terahertz spectroscopy with quantumcascade lasers, J. Appl. Phys. 125, 151401 (2019)

[3] J. Faist et al., Quantum Cascade Laser, Science 264, 553 (1994)

[4] B. S. Williams, Terahertz quantumcascade lasers, Nat. Photonics 1, 517 (2007)

[5] C. Jirauschek et al., Modeling techniques for quantum cascade lasers, Appl. Phys. Rev. 1, 011307 (2014)

[6] L. Schrottke et al., High-performance GaAs/AlAs terahertz quantum-cascade lasers for spectroscopic applications, IEEE Trans. Terahertz Sci. Technol. 10, $133(2020)$
[7] H. Richter et al., 4.7-THz local oscillator for the GREAT heterodyne spectrometer on SOFIA, IEEE Trans. Terahertz Sci. Technol. 5, 539 (2015)

[8] L. Rezac et al., First detection of the $63 \mu \mathrm{m}$ atomic oxygen line in the thermosphere of Mars with GREAT/SOFIA, Astron. Astrophys. 580, L10 (2015)

[9] M. S. Vitiello et al., Quantum-limited frequency fluctuations in a terahertz laser, Nat. Photonics 6, 525 (2012)

[10] D. J. Hayton et al., Phase locking of a 3.4 THz third-order distributed feedback quantum cascade laser using a roomtemperature superlattice harmonic mixer, Appl. Phys. Lett. 103, 051115 (2013)

[11] S. Bartalini et al., Frequencycomb-assisted terahertz quantum cascade laser spectroscopy, Phys. Rev. X 4, 021006 (2014)
[12] J. Freeman et al., Injection locking of a terahertz quantum cascade laser to a telecommunications wavelength frequency comb, Optica 4, 1059 (2017)

[13] M. Hempel et al., Fast continuous tuning of terahertz quantum-cascade lasers by rear-facet illumination, Appl. Phys. Lett. 108, 191106 (2016)

[14] T. Alam et al., Wideband, highresolution terahertz spectroscopy by light-induced frequency tuning of quantum-cascade lasers, Opt. Express 27, 5420 (2019)

[15] M. Wienold et al., Doppler-free spectroscopy with a terahertz quantumcascade laser, Opt. Express 26, 6692 (2018)

[16] T. Hagelschuer et al., Real-time gas sensing based on optical feedback in a terahertz quantum-cascade laser, Opt. Express 25, 30203 (2017) 